\title{
HOMOTOPY TYPE FINITENESS THEOREMS FOR CERTAIN PRECOMPACT FAMILIES OF RIEMANNIAN MANIFOLDS
}

\author{
TAKAO YAMAGUCHI
}

(Communicated by David G. Ebin)

\begin{abstract}
In this paper, we consider a precompact family of Riemannian manifolds with respect to the Hausdorff distance, and prove the homotopy type finiteness of elements in the family. This is an extension in the homotopy type version of the Cheeger and Weinstein finiteness theorems.
\end{abstract}

The notion of Hausdorff distance between Riemannian manifolds was introduced by Gromov [11], and has played an important role in global Riemannian geometry. In [11], he proved the so-called precompactness theorem:

TheOREM (GROMOV). For given $m, \Lambda, D$, the family of compact Riemannian m-manifolds $M$, whose curvatures $\operatorname{Ric}_{M}$ and diameters ${ }^{\prime}{ }_{M}$ satisfy $\operatorname{Ric}_{M} \geq$ $-(m-1) \Lambda^{2}$ and $d_{M} \leq D$, is precompact with respect to the Hausdorff distance.

It was also proved in [11] that the first Betti numbers of manifolds in the above family are uniformly bounded in terms of $m, \Lambda, D$, and that the convergences with repsect to the Hausdorff distance and the Lipschitz distance coincide on the family of compact $m$-mainfolds $M$ with bounded sectional curvatures $\left|K_{M}\right| \leq \Lambda^{2}$, volumes $\operatorname{vol}_{M} \leq V$ and injectivity radii $i_{M} \geq \varepsilon$ for given positive constants $\Lambda, V, \varepsilon$. From the last result, the Cheeger and Weinstein finiteness theorems $[\mathbf{4}, 20]$ are derived. For related results, see $[\mathbf{6}, \mathbf{7}, \mathbf{8}, \mathbf{1 4}, \mathbf{1 6}]$.

The purpose of the present paper is to prove the homotopy type finiteness for any precompact family of complete manifolds whose contractibility radii are uniformly bounded below by a positive constant. The contractibility radius $c_{M}$ of a complete manifold $M$ is defined as the supremum of $r$ such that every metric ball of radius $r$ contains no critical points of the distance function from the center. For the precise definition, see $\S 1$. Thus $c_{M}$ is greater than or equal to the injectivity radius $i_{M}$.

Let $\mathfrak{M}$ be a precompact family of complete $m$-manifolds with respect to the Hausdorff distance $d_{H}$. For given $R>0$, we set $\mathfrak{M}_{R}=\left\{M \in \mathfrak{M} ; c_{M} \geq R\right\}$. In this situation, we shall prove the following

THEOREM 1. The set of homotopy types of manifolds in $\mathfrak{M}_{R}$ is finite.

Theorem 1 is a direct consequence of the following theorem.

THEOREM 2. Let $M, M^{\prime}$ be complete m-manifolds with $c_{M}, c_{M^{\prime}} \geq R$. If

$$
d_{H}\left(M, M^{\prime}\right)<R / 25 m
$$

then $M$ has the same homotopy type as $M^{\prime}$.

Received by the editors October $1,1986$.

1980 Mathematics Subject Classification (1985 Revision). Primary 53C20; Secondary 53C22.

(C) 1988 American Mathematical Society $0002-9939 / 88 \$ 1.00+\$ .25$ per page 
From Theorem 1, we have immediately

COROLlaRY 1. For given $m$ and positive numbers $\Lambda, D, R$, the set of homotopy types of compact $m$-manifolds $M$ with $\operatorname{Ric}_{M} \geq-(m-1) \Lambda^{2}, d_{M} \leq D$ and $c_{M} \geq R$ is finite.

COROLlaRY 2. For given $m$ and $L$, the set of homotopy types of compact $m$ manifolds $M$ with bounded embolic volumes $\operatorname{vol}_{M} / i_{M}^{m} \leq L$ is finite.

COROLlaRY 3. For given $m, \Lambda$ and $R \in(\pi / 2, \pi]$, the set of homotopy types of compact $m$-manifolds $M$ with $\operatorname{Ric}_{M} \geq(m-1), K_{M} \geq-\Lambda^{2}$ and $\operatorname{vol}_{M} \geq \alpha(m, R)$ is finite, where $\alpha(m, R)$ is the volume of the $R$-ball in the unit $m$-sphere.

Corollaries 1 and 2 could be thought of as extensions of the Cheeger and Weinstein finiteness theorems, though the results tell us only homotopy types. Corollary 3 gives an affirmative answer to the problem proposed by Shiohama [19], who first considered the notion of contractibility radius by specializing the notion of content of a metric ball introduced by Gromov [9]. It shold also be noted that in the case when all manifolds in the family $\mathfrak{M}$ are compact, the number of homotopy types in $\mathfrak{M}_{R}$ given by Theorem 1 can be estimated explicity in terms of an invariant depending of $R$ and $\mathfrak{M}$.

1. Uniform contractibility. In this section, we provide a technical tool needed in the proof of Theorem 2. We first recall the definition of critical points of distance functions. For a fixed point $x$ in a complete manifold $M$, consider the distance function $d_{x}: d_{x}(y)=d(x, y)$. A point $y(\neq x)$ is called a critical point of $d_{x}$ if for every nonzero tangent vector $v \in T_{y} M$, there is a minimal geodesic $\gamma$ from $y$ to $x$ such that $v$ and $\gamma$ make an angle at most $\pi / 2$. Obviously, a critical point of $d_{x}$ lies in the cut locus of $x$. The contractibility radius $c_{x}$ at $x$ is defined by $c_{x}=\inf \left\{d(x, y) ; y\right.$ a critical point of $\left.d_{x}\right\}$. Since the (open) metric ball $B\left(x, c_{x}\right)$ around $x$ of radius $c_{x}$ contains only noncritical points of $d_{x}$, for every $y_{0}$ in the ball there is a neighborhood $U$ of $y_{0}$ and a smooth vector field $t$ on $U$ such that for each $y \in U, t(y)$ and every minimal geodesic from $y$ to $x$ make an acute angle. We call such a vector field $t$ gradient-like for $d_{x}$. From this observation, an easy Morse theoretic argument implies the contractibility of $B\left(x, c_{x}\right)$ (cf. Gromov [11, 1.1]. Clearly $c_{x}$ is lower semicontinuous in $x$ and not less than the injectivity radius at $x$. The contractibility radius $c_{M}$ of $M$ is defined as the infimum of $c_{x}$ when $x$ runs over $M$.

Now observe that every two points $x$ and $y$ with $d(x, y)<i_{M}$ can be joined by a unique minimal geodesic $\gamma_{x, y}:[0,1] \rightarrow M$ and that $\gamma_{x, y}(t)$ is smoothly depending on $x, y$ and $t$. This fact is extended in a sense to the contractibility radius in the following way. For every $\varepsilon>0$, put $\Omega_{\varepsilon}=\left\{(x, y) \in M \times M ; d(x, y)<c_{M}-\varepsilon\right\}$.

PROPOSITION 1.1. There is a smooth map $h: \Omega_{\varepsilon} \times[0,1] \rightarrow M$ satisfying the following conditions:

(1) $h(x, y, 0)=y, h(x, y, 1)=x$.

(2) The function $t \rightarrow d_{x}(h(x, y, t))$ is strictly decreasing.

We call such an $h$ a uniform retraction of contractible metric balls. For the proof, we prepare two lemmas. We denote by $\Delta_{S}$ the diagonal set of $S . \pi: U M \rightarrow M$ denotes the unit tangent bundle of $M$. For a metric $R$-ball $B$ and $\delta>0, \delta B$ denotes the concentric ball of radius $\delta R$. 
LEMMA 1.2. Let $B$ be a convex ball around $x$ in $M$. For a sufficiently small neighborhood $O \subset B$ of $x$, there is a smooth map $X: O \times \bar{B}-\Delta_{0} \rightarrow U M, X_{z}(y)=$ $X(z, y)$, with the following properties:

(1) $\pi \circ X(z, y)=y$, that is, $X_{z}$ is a smooth vector field on $B-\{z\}$.

(2) For every point $y$ on a neighborhood of the boundary $\partial B, X_{z}(y)$ is tangent to the minimal geodesic from $y$ to $x$.

(3) For every point $y$ on a neighborhood of $\frac{1}{2} \bar{B}, X_{z}(y)$ is tangent to the minimal geodesic from $y$ to $z$.

(4) For every point $z$ in $O, X_{z}$ is gradient-like for $d_{z}$.

ProOF. Every point $y$ in $\bar{B}-\frac{1}{2} B$ lies in the minimal geodesic from a point $y^{\prime}$ in $\partial B$ to $x$. By the diffeomorphism of $\bar{B}-\frac{1}{2} B$ onto $\partial B \times[0,1], y \rightarrow\left(y^{\prime}, t\right), t=$ $2 d\left(y, y^{\prime}\right) / \delta$ ( $\delta$ is the radius of $B$ ), we identify $y$ and $\left(y^{\prime}, t\right)$. Take a monotone function $f:[0,1] \rightarrow[0,1]$ such that $f([0, \alpha))=0$ and $f((1-\alpha, 1])=1$ for some $\alpha>0$. For every $z \in O$, let $\gamma_{x, z}:[0,1] \rightarrow M$ be the minimal geodesic from $x$ to $z$. We define $X(z, y)$ as the unit initial velocity of the minimal geodesic from $y=\left(y^{\prime}, t\right)$ to $\gamma_{x, y}(f(t))$. X can be extended to $O \times \bar{B}-\Delta_{0}$ so as to satisfy (1), (2), (3) and (4) if $O$ is taken sufficiently small.

LEMMA 1.3. For every large positive number $R^{\prime}$ and every small $\varepsilon>0$, we set $R=\min \left\{c_{M}, R^{\prime}\right\}$ and $W_{\varepsilon}=\{(x, y) \in M \times M ; 0 \neq d(x, y)<R-\varepsilon\}$. Then there is a smooth map $X: W_{\varepsilon} \rightarrow U M, X_{x}(y)=X(x, y)$, such that

(1) $\pi \circ X(x, y)=y$,

(2) For every $x \in M, X_{x}$ is a gradient-like vector field of $d_{x}$ on $B(x, R-\varepsilon)$.

PROOF. We fix a point $x$ for a mement. From the definition of critical points, for each $y \in B(x, R)$ we can find a neighborhood $O_{y}(x)$ of $x$, a neighborhood $U(y)$ of $y$ and a smooth vector field $t_{y}$ on $U(y)$ such that $t_{y}$ is a gradient-like vector field of $d_{z}$ for all $z \in O_{y}(x)$. Take a small convex ball $B$ around $x$ and choose $y_{1}, \ldots, y_{k}$ with $B(x, R)-B \subset \bigcup_{i=1}^{k} U\left(y_{i}\right)$, where $U\left(y_{i}\right), O_{y_{i}}(x)$ and $t_{y_{i}}$ are chosen so as to have the above properties. Putting $X_{x}^{\prime}=\sum \lambda_{i} t_{y_{i}} /\left|\sum \lambda_{i} t_{y_{i}}\right|$, where $\left\{\lambda_{i}\right\}$ is a partition of unity dominated with $\left\{U\left(y_{i}\right)\right\}$, we obtain a vector field $X_{x}^{\prime}$ on $B(x, R)-B$ such that $X_{x}^{\prime}$ is a gradient-like vector field of $d_{z}$ for all $z$ in $O(x)=\bigcap_{i=1}^{k} O_{y_{i}}(x)$. We may assume that $O(x)$ is included in $B(x, \varepsilon)$ and that for every $y$ in a neighborhood of $\partial B, X_{x}^{\prime}(y)$ is tangent to the velocity vector of the minimal geodesic from $y$ to $x$. By Lemma 1.2, we can construct a smooth map $X^{(x)}: O(x) \times B(x, R)-\Delta_{O(x)} \rightarrow U M$ with the following properties:

(i) For every $z \in O(x), X_{z}^{(x)}$ is an extension of $X_{x}^{\prime}: B(x, R)-B \rightarrow U M$.

(ii) For every point $y$ on a neighborhood of $\frac{1}{2} \bar{B}, X_{z}^{(x)}(y)$ is tangent to the minimal geodesic from $y$ to $z$.

(iii) For every $z \in O(x), X_{z}^{(x)}$ is a gradient-like vector field of $d_{z}$ on $B(x, R)$, and hence on $B(z, R-\varepsilon)$.

Now we move $x$ on $M$, and take a locally finite covering $\left\{O\left(x_{j}\right)\right\}$ of $M$ and a partition of unity $\left\{u_{j}\right\}$ dominated with $\left\{O\left(x_{j}\right)\right\}$. The required smooth map $X$ is defined by

$$
X_{x}(y)=\sum \mu_{j}(x) X_{x}^{\left(x_{j}\right)}(y) /\left|\sum \mu_{j}(x) X_{x}^{\left(x_{j}\right)}(y)\right| .
$$

Proof OF Proposition 1.1. Assume first $c_{M}<\infty$. For $R=c_{M}$, take a smooth map $X: W_{\varepsilon} \rightarrow U M$ as in Lemma 1.3. For $(x, y) \in W_{\varepsilon}=\Omega_{\varepsilon}-\Delta_{M}$, let 
$\phi_{x, y}(t)$ be the integral curve of $X_{x}$ with $\phi_{x, y}(0)=y$, and let $t(x, y)$ be the time at which $\phi_{x, y}$ reaches $x ; \phi_{x, y}(t(x, y))=x$. The uniform retraction $h$ is given by $h(x, y, t)=\phi_{x, y}(t \cdot t(x, y))$. In the case $c_{M}=\infty$, an obvious iterated precedure in Lemma 1.3 for an increasing sequence of concentric balls would supply $h$.

2. Proof Theorem 2. We recall the definition of the Hausdorff distance. For details, see [11, Chapters $3,5,8]$. For subsets $A$ and $B$ in a metric space $Z$, we denote by $d_{H}^{Z}(A, B)$ the infimum of positive $\varepsilon$ such that the $\varepsilon$-neighborhoods of $A$ and $B$ include $B$ and $A$ respectively. The Hausdorff distance between two metric spaces $X$ and $Y$ is by definition

$$
d_{H}(X, Y)=\inf _{Z, f, g} d_{H}^{Z}(f(X), g(Y))
$$

where $Z$ is a metric space and $f: X \rightarrow Z$ and $g: Y \rightarrow Z$ are isometric imbeddings.

PROOF OF THEOREM 2. For given $R>0$, we set $\delta=R / 25 m$ and $r=R / 100 m$. Let $M$ and $M^{\prime}$ be complete $m$-manifolds such that $c_{M}, c_{M}, \geq R$ and $d_{H}\left(M, M^{\prime}\right)<$ $\delta$. Let $\left\{B_{i}\right\}_{i=1, \ldots, N}(N \leq \infty)$ be a locally finite covering of $M$ by $r$-balls and let $p_{i}$ be the center of $B_{i}$. By the definition of Harsdorff distance, there are $p_{i}^{\prime}$ in $M^{\prime}$ such that $\left|d\left(p_{i}, p_{j}\right)-d\left(p_{i}^{\prime}, p_{j}^{\prime}\right)\right|<2 \delta$, and that the balls $B_{i}^{\prime}$ around $p_{i}^{\prime}$ of radius $r^{\prime}=r+2 \delta$ cover $M^{\prime}$. Let $\left\{f_{i}\right\}$ and $\left\{f_{i}^{\prime}\right\}$ be partitions of unity dominated with $\left\{B_{i}\right\}$ and $\left\{B_{i}^{\prime}\right\}$ respectively such that $f_{i}>0$ on $B_{i}, f_{i}=0$ outside $B_{i}$ and $f_{i}^{\prime}>0$ on $B_{i}^{\prime}, f_{i}^{\prime}=0$ outside $B_{i}^{\prime}$. We define mappings $f: M \rightarrow \mathbf{R}^{N}, f^{\prime}: M^{\prime} \rightarrow \mathbf{R}^{N}$ by

$$
f(x)=\left(f_{1}(x), \ldots, f_{N}(x)\right), \quad f^{\prime}(y)=\left(f_{1}^{\prime}(y), \ldots, f_{N}^{\prime}(y)\right) .
$$

Let $K$ and $K^{\prime}$ be the nerves of the coverings $\left\{B_{i}\right\}$ and $\left\{B_{i}^{\prime}\right\}$ respectively. They are realized as subcomplexes of the unit simplex $\Sigma=\left\{\left(x_{1}, \ldots, x_{N}\right) ; \sum x_{i}=1, x_{i} \geq 0\right\}$. Let $L$ be the union of $K$ and $K^{\prime}$, which is also a subcomplex of $\Sigma$. Thus $f$ and $f^{\prime}$ can be considered as mappings into $K \subset L$ and $K^{\prime} \subset L$ respectively. For $v_{i}=(0, \ldots, \check{1}, \ldots, 0) \in \mathbf{R}^{N}(1$ in the $i$ th position $)$, let $\Delta^{k}\left(i_{0}, \ldots, i_{k}\right), i_{0}<\cdots<i_{k}$, be the $k$-simplex of $\Sigma$ spanned by $v_{i_{0}}, \ldots, v_{i_{k}}$. We define a mapping $f_{m}$ of $M$ into the $m$-skeleton $K^{m}$ of $K$ inductively as follows: For $x \in M, f(x)$ is contained in a $k$-simplex $\Delta^{k}$ of $K$, where we may assume $k>m$. Then take a point $p$ in $\Delta^{k}-f(M)$, and denote by $f_{k-1}(x)$ the image of $f(x)$ under the radial projection from $p$ onto the boundary $\partial \Delta^{k}$. We repeat this process to obtain the mapping $f_{m}: M \rightarrow K^{m}$. Similarly, $f_{m}^{\prime}: M^{\prime} \rightarrow\left(K^{\prime}\right)^{m}$ is defined. Conversely, we construct a mapping $g: L^{m}=K^{m} \cup\left(K^{\prime}\right)^{m} \rightarrow M$ inductively as follows: We first put $g\left(v_{i}\right)=p_{i}$ on $L^{0}$ and assume a mapping $g: L^{k-1} \rightarrow M, k-1<m$, is defined so as to satisfy $d\left(g(x), p_{i_{\alpha}}\right)<(4 r+12 \delta) s$ for every simplex $\Delta^{s}=\Delta^{s}\left(i_{0}, \ldots, i_{s}\right)$ in $L^{k-1}$ and for every $x \in \Delta^{s}$ and $0 \leq \alpha \leq s$. Now for a $k$-simplex $\Delta^{k}=\Delta^{k}\left(i_{0}, \ldots, i_{k}\right)$ of $L$, let $b_{\Delta} k$ denote the barycenter of $\Delta^{k}$, and define $g$ on $\Delta^{k}$ by $g\left((1-t) x+t b_{\Delta} k\right)=$ $h\left(p_{i_{0}}, g(x), t\right)$, where $x \in \partial \Delta^{k}, 0 \leq t \leq 1$, and $h$ is a uniform retraction given in Proposition 1.1. We have to check that $h\left(p_{i_{0}}, g(x), t\right)$ is well defined. Choose a vertex $v_{i_{\alpha}}$ in the open simplex containing $x$, and notice that if $\Delta^{k} \in K$, then $d\left(p_{i_{0}}, p_{i_{\alpha}}\right)<2 r$ and if $\Delta^{k} \in K^{\prime}$, then $d\left(p_{i_{0}}, p_{i_{\alpha}}\right) \leq d\left(p_{i_{0}}^{\prime}, p_{i_{\alpha}}^{\prime}\right)+2 \delta<2 r+6 \delta$. It follows that

$$
\begin{aligned}
d\left(p_{i_{0}}, g(x)\right) & \leq d\left(p_{i_{0}}, p_{i_{\alpha}}\right)+d\left(p_{i_{\alpha}}, g(x)\right) \\
& \leq(2 r+6 \delta)+(4 r+12 \delta)(k-1) \ll R .
\end{aligned}
$$


Thus $h\left(p_{i_{0}}, g(x), t\right)$ is well defined, and we have for every $0 \leq \beta \leq k$,

$$
\begin{aligned}
d\left(h\left(p_{i_{0}}, g(x), t\right), p_{i_{\beta}}\right) & \leq d\left(h\left(p_{i_{0}}, g(x), t\right), p_{i_{0}}\right)+d\left(p_{i_{0}}, p_{i_{\beta}}\right) \\
& \leq d\left(p_{i_{0}}, g(x)\right)+d\left(p_{i_{0}}, p_{i_{\beta}}\right) \\
& \leq(2 r+6 \delta)+(4 r+12 \delta)(k-1)+(2 r+6 \delta) \\
& =(4 r+12 \delta) k .
\end{aligned}
$$

We have just constructed the mapping $g: L^{m} \rightarrow M$. Similarly, a mapping $g^{\prime}: L^{m} \rightarrow$ $M^{\prime}$ is also constructed so as to satisfy $d\left(g^{\prime}(x), p_{i_{\alpha}}^{\prime}\right)<(4 r+8 \delta) s$ for every simplex $\Delta^{s}=\Delta^{s}\left(i_{0}, \ldots, i_{s}\right)$ in $L^{m}$ and for every $x \in \Delta^{s}$ and $0 \leq \alpha \leq s$. We set $\Phi=$ $g^{\prime} \circ f_{m}, \Phi^{\prime}=g \circ f_{m}^{\prime}$. To show that $\Phi$ is a homotopy equivalence with $\Phi^{\prime}$ as a homotopy inverse, we estimate the $C^{0}$ distance between $\Phi^{\prime} \circ \Phi$ and the identity. For a fixed $x \in M$, let $\Delta^{k}=\Delta^{k}\left(i_{0}, \ldots, i_{k}\right)$ and $\Delta^{s}=\Delta^{s}\left(j_{0}, \ldots, j_{s}\right)$ be the simplices of $L^{m}$ with $f_{m}(x) \in \Delta^{k}, f_{m}^{\prime} \circ \Phi(x) \in \Delta^{s}$. From the construction, we see for every $0 \leq \alpha \leq k$ and $0 \leq \beta \leq s$,

$$
\begin{gathered}
d\left(x, p_{i_{\alpha}}\right)<r, \quad d\left(\Phi(x), p_{j_{\beta}}^{\prime}\right)<r^{\prime} \\
d\left(\Phi(x), p_{i_{\alpha}}^{\prime}\right)<(4 r+8 \delta) k \leq(4 r+8 \delta) m, \\
d\left(\Phi^{\prime} \circ \Phi(x), p_{j_{\beta}}\right)<(4 r+12 \delta) s \leq(4 r+12 \delta) m .
\end{gathered}
$$

It follows that

$$
\begin{aligned}
d\left(x, \Phi^{\prime} \circ \Phi(x)\right) & \leq d\left(x, p_{i_{\alpha}}\right)+d\left(p_{i_{\alpha}}, p_{j_{\beta}}\right)+d\left(p_{j_{\beta}}, \Phi^{\prime} \circ \Phi(x)\right) \\
& \leq r+d\left(p_{i_{\alpha}}, p_{j_{\beta}}\right)+(4 r+12 \delta) m,
\end{aligned}
$$

where $d\left(p_{i_{\alpha}}, p_{j_{\beta}}\right)<d\left(p_{i_{\alpha}}^{\prime}, p_{j_{\beta}}^{\prime}\right)+2 \delta$ and $d\left(p_{i_{\alpha}}^{\prime}, p_{j_{\beta}}^{\prime}\right) \leq d\left(p_{i_{\alpha}}^{\prime}, \Phi(x)\right)+d\left(\Phi(x), p_{j_{\beta}}^{\prime}\right) \leq$ $(4 r+8 \delta) m+r^{\prime}$. This implies

$$
d\left(x, \Phi^{\prime} \circ \Phi(x)\right)<(8 m+2) r+(20 m+4) \delta<R .
$$

Similarly, we get $d\left(y, \Phi \circ \Phi^{\prime}(y)\right)<(8 m+2) r+(20 m+4) \delta<R$ for every $y$ in $M^{\prime}$. Thus $M$ has the same homotopy type as $M^{\prime}$ by Proposition 1.1.

3. Precompact families. Let $\mathfrak{M}$ be a precompact family of complete $m$ manifolds with repsect to the Haudorff distance. This means for every $\varepsilon>0$, there exist finitely many elements $M_{1}, \ldots, M_{k(\varepsilon)}$ in $\mathfrak{M}$ such that for every $M$ in $\mathfrak{M}, d_{H}\left(M, M_{i}\right)<\varepsilon$ for some $1 \leq i \leq k(\varepsilon)$. Hence Theorem 2 shows that for a given $R>0$, the number of homotopy types in $\mathfrak{M}_{R}$ does not exceed $k(\delta)$ for $\delta=R / 25 m$.

In the case when all manifolds in $\mathfrak{M}$ are compact, we estimate the number $k(\delta)$ explicitly as follows: For $M$ in $\mathfrak{M}$ and $\varepsilon>0$, let $N(\varepsilon, M)$ be the maximal number of disjoint $\varepsilon$-balls in $M$. The precompactness of $\mathfrak{M}$ implies that the supremum $N_{\mathfrak{M}}(\varepsilon)=\sup \{N(\varepsilon, M) ; M \in \mathfrak{M}\}$ is finite. Put $N=N_{\mathfrak{M}}(\delta / 2)$. Notice $d_{M} \leq 2 \delta N$ for all $M$ in $\mathfrak{M}$. Then using the Dirichlet drawer principle and the argument in [11, 5.2], we get $k(\delta)<N^{4}$. Thus we have Theorem 1 with the explicit bound.

THEOREM $1^{\prime}$. In the case when all manifolds in $\mathfrak{M}$ are compact, the number of homotopy types in $\mathfrak{M}_{R}$ does not exceed $N_{\mathfrak{M}}(R / 50 m)^{4}$.

We should remark that in the compact case, it is already known, from the Topological Lemma of Gromov [9] that the Betti number sum $\sum_{i=0}^{m} b_{i}(M ; F)$ with any coefficient field $F$ does not exceed $(m+1) 2^{N_{\mathfrak{M}}\left(R /\left(5 \cdot 10^{m+1}\right)\right)}$ for every $M$ in $\mathfrak{M}_{R}$. 
Next we consider the following families of compact $m$-manifolds $M$ :

$$
\begin{aligned}
& \mathfrak{M}^{1}=\left\{M ; \operatorname{Ric}_{M} \geq-(m-1) \Lambda^{2}, d_{M} \leq D\right\}, \\
& \mathfrak{M}^{2}=\left\{M ; \operatorname{vol}_{M} \leq V, i_{M} \geq R\right\} .
\end{aligned}
$$

The volume comparison theorem due to Bishop and Gromov [11., 5.3], implies $N_{\mathfrak{M}^{1}}(R / 50 m) \leq b_{\Lambda}(D) / b_{\Lambda}(R / 50 m)$, where $b_{\Lambda}(\varepsilon)$ is the volume of an $\varepsilon$-ball in the simply connected space of constant curvature $-\Lambda^{2}$. On the other hand, the volume estimate due to Croke [5, Proposition 14] states that for each $r \leq \frac{1}{2} i_{M}$, the volume of every $r$-ball is greater than $c_{1}(m) r^{m}$, where $c_{1}(m)$ depends only on $m$. From this, the precompactness of $\mathfrak{M}^{2}$ and the following estimate are derived: $N_{\mathfrak{m}^{2}}(R / 50 m) \leq$ $c_{2}(m)^{m}\left(V / R^{m}\right), c_{2}(m)=(50 m)^{m} / c_{1}(m)$. Thus we restate Corollaries 1 and 2 with the explicit estimates:

COROLLARY $1^{\prime}$. The number of homotopy types of compact m-manifolds $M$ with

$$
\operatorname{Ric}_{M} \geq-(m-1) \Lambda^{2}, \quad d_{M} \leq D \quad \text { and } \quad c_{M} \geq R
$$

does not exceed $\left(b_{\Lambda}(D) / b_{\Lambda}(R / 50 m)\right)^{4}$.

COROLlaRY $2^{\prime}$. The number of homotopy types of compact $m$-manifolds $M$ with $\operatorname{vol}_{M} / i_{M}^{m} \leq L$ does not exceed $c(m) L^{4}$, where $c(m)$ depends only on $m$.

Finally, for given $m, \Lambda$ and $R \in(\pi / 2, \pi]$, we consider the subfamily $\mathfrak{M}^{3}$ of $\mathfrak{M}^{1}$ consisting of certain manifolds with positive Ricci curvature:

$$
\mathfrak{M}^{3}=\left\{M ; \operatorname{Ric}_{M} \geq(m-1), K_{M} \geq-\Lambda^{2}, \operatorname{vol}_{M} \geq \alpha(m, R)\right\} .
$$

Notice $d_{M} \leq \pi$ and $M$ is simply connected for every $M$ in $\mathfrak{M}^{3}$ by the classical theorems of Myers [15] and Biship [3]. A uniform estimate on the contractibility radii $c_{M}$ for all $M$ in $\mathfrak{M}^{3}$ was given by Shiohama [19]. Together with this, Corollary $1^{\prime}$ would provide an explicit estimate on the number of homotopy types in $\mathfrak{M}^{3}$. Notice, in [19], the restriction $R \in(2 \pi / 3, \pi]$ was needed for the uniform estimate on $c_{M}$, but this is not essential. A similar proof applies to our situation. It will be easily seen that the family $\bigcup_{R>\pi / 2} \mathfrak{M}^{3}$ contains the following manifolds with the standard Einstein metrics: For $m \leq 8$, for example,

$$
\begin{aligned}
& m=2,3: S^{2}, S^{3} ; \quad m=4: S^{4}, P^{2}(\mathbf{C}), S^{2} \times S^{2} ; \quad m=5: S^{5}, S^{2} \times S^{3} ; \\
& m=6: S^{6}, P^{3}(\mathbf{C}), S^{2} \times S^{4}, S^{3} \times S^{3}, S^{2} \times P^{2}(\mathbf{C}) ; \\
& m=7: S^{7}, S^{2} \times S^{5}, S^{3} \times S^{4}, S^{3} \times P^{2}(\mathbf{C}) ; \\
& m=8: S^{8}, P^{4}(\mathbf{C}), P^{2}(\mathbf{H}), S^{2} \times S^{6}, S^{3} \times S^{5}, S^{4} \times S^{4}, S^{4} \times P^{2}(\mathbf{C}), \\
& \quad S^{2} \times S^{2} \times S^{4}, S^{2} \times S^{3} \times S^{3}, S^{2} \times S^{2} \times S^{2} \times S^{2} .
\end{aligned}
$$

The sphere theorem due to Shiohama [19] (see also Itokawa [13]) shows that when $R$ is close to $\pi$, the set of homeomorphism classes in $\mathfrak{M}^{3}$ consists of the single $S^{m}$.

On the other hand, according to the Berger isoembolic inequality [1],

$$
\operatorname{vol}_{M} / i_{M}^{m} \geq \operatorname{vol}_{S^{m}} / i_{S^{m}}^{m},
$$

where $S^{m}$ is a round sphere and the equality holds if and only if $M$ is a round sphere. Corollary 2 will support the following problem.

PROBLEM. Is there a positive constant $\delta(m)$ depending only on $m$ such that if $\operatorname{vol}_{M} / i_{M}^{m}<\operatorname{vol}_{S^{m}} / i_{S^{m}}^{m}+\delta(m)$, then $M$ is homeomorphic to $S^{m}$ ? 


\section{REFERENCES}

1. M. Berger, Une borne inférieure pour le volume d'une variété riemannienne et fonction du rayon d'injectivite, Ann. Inst. Fourier (Grenoble) 30 (1980), 259-265.

2. - Recent trends in riemannian geometry, preprint, 1982.

3. R. Biship, A relation between volume, mean curvature, and diameter, Notices Amer. Math. Soc. 10 (1963), 364.

4. J. Cheeger, Finiteness theorems for Riemannian manifolds, Amer. J. Math. 92 (1970), 61-74.

5. C. Croke, Some isoperimetric inequalities and eigenvalue estimates, Ann. Sci. Ecole Norm. Sup. 13 (1980), 419-435.

6. K. Fukaya, A boundary of the set of Riemannian manifolds with bounded curvatures and diameters, preprint, 1985.

7. S. Gallot, Inégalités isopérimétriques, courbure de Ricci et invariants géométriques, C.R. Acad. Sci. Paris 296 (1983), 333-336 et 365-368.

8. R. E. Greene and H. Wu, Lipschitz convergence of Riemannian manifolds, preprint, 1985.

9. M. Gromov, Curvature, diameter and Betti numbers, Comment. Math. Helv. 56 (1981), 179195.

10. _ Volume and bounded cohomology, Inst. Hautes Etudes Sci. Publ. Math. 56 (1983), 223230.

11. __ Structures métriques pour les variété riemanniennes, rédigé par J. Lafontaine et P. Pansu, Cedic/Fernand Nathan, Paris, 1981.

12. K. Grove and K. Shiohama, A generalized sphere theorem, Ann. of Math. (2) 106 (1977), 201-211.

13. Y. Itokawa, The topology of certain Riemannian manifolds with positive Ricci curvature, J. Differential Geom. 18 (1983), 151-156.

14. A. Katsuda, Gromov's convergence theorem and its application, Nagoya Math. J. 100 (1985), 11-48.

15. S. B. Myers, Riemannian manifolds with positive mean curvature, Duke Math. J. 8 (1941), 401-404.

16. S. Peters, Cheeger's finiteness theorem for diffeomorphism classes of Riemannian manifolds, J. Reine Angew. Math. 349 (1984), 77-82.

17. G. De Rham, Complexes à automorphismes et homémorphie différentiable, Ann. Inst. Fourier (Grenoble) 2 (1950), 51-67.

18. T. Sakai, Comparison and finiteness theorems in Riemannian geometry, Geometry of Geodesics and Related Topics, Advanced Studies in Pure Math., vol 3, 1984, pp. 125-181.

19. K. Shiohama, A sphere theorem for manifoldss of positive Ricci curvature, Trans. Amer. Math. Soc. 275 (1983), 811-819.

20. A. Weinstein, On the homotopy types of positively pinched manifolds, Arch. Math. 18 (1967), 523-524.

21. T. Yamaguchi, Locally geodesically quasiconvex functions on complete Riemannian manifolds, Trans. Amer. Math. Soc. 298 (1986), 301-330.

Department of Mathematics, Faculty of Science and Engineering, Saga UNIVERSITY, SAGA 840, JAPAN 